\title{
Pregnancy-related microchimerism unknown pathophysiological effects
}

\author{
David Davies and Osman Demirhan* \\ Adana City Training and Research Hospital, Beyazevler neighborhood, Adana, Turkey
}

\section{Introduction}

In genetics, microchimerism (Mc) is defined by the presence within an individual of a low level of cells or DNA derived from a genetically different individual. Pregnancy is the most common and natural cause of chimerism, and bi-directional trafficking of hematopoietic cells occurs through the placenta. During pregnancy there is bidirectional exchange of cells between the pregnant woman and her fetus through the placenta, potentially leading to the establishment of microchimerism in both the woman and the fetus. Therefore, we are all born as microchimera. Since all humans are born as microchemera, we must accept physiologically acceptable feto-maternal microchimerism. The presence of fetal cells in maternal circulation has been known for a century. However, the biological effects of this formation have not been largely explored. Fetal microchimeric cells (FMcCs) stay in the maternal bone marrow for many years after birth and migrate to blood and tissues. The emerging situation has not yet clarified how the cells migrate to the new environment and how it affects their ability to sustain their lives, integrate and differentiate in this environment. Although there are many unanswered questions it is thought that chimerism has an important role in human health. For many years, the clinical effects of maternal microchimeric cells (MMcCs) in organ repair and cancer therapy have just begun to be understood. While the mission of chimerism is straightforward, the subject is profound. Chimerism carries the potential for disease as well as for health benefits, provides us with a major experimental tool to deepen our understanding in biology.

The exact role of fetal microchimerism (FMc) remains, at least in part, an unsolved topic. However, three hypotheses have been formulated over the past decade. The maternal stress and infections in pregnancy affect fetal neurodevelopment and increase the risk for neurological or psychiatric disorders in future life of the fetus. The persistence of $\mathrm{FMcCs}$ in maternal tissues could lead to a response similar to that of the graft-versus-host disease (GVHD), with chronic inflammatory responses that lead to tissue damage. The other hypothesis suggests that FMc could have a protective role in repairing damaged tissues, in cancer surveillance and in the control of viral infections. Finally, some argue that FMcCs are simply an incidental by product of pregnancy without biological significance. However, we suggest that feto-maternal microchimerism should be considered as a physiological event. However, the phenomenon of fetal-maternal microchimerism (F-MMc) inspires numerous questions. In this review, we think that Mc is a physiological phenomenon. This phenomenon can also turn into physiopathology under inappropriate conditions. We describe in detail below the possible pathophysiological roles of the F-MMcCs.

\section{Microchimerism physiopathology}

Pregnancy is the most common and natural cause of chimerism, and bi-directional trafficking of hematopoietic cells occurs through the placenta. During pregnancy there is bidirectional exchange of cells between the pregnant woman and her fetus through the placenta, potentially leading to the establishment of microchimerism in both the woman and the fetus. This cell transfer occurs physiologically. McCs that remain within physiological limits may also be harmful. Emerging evidence suggests it has been shown that fetal microchimeric cells may be effective in rejuvenating progenitor cell source, repairing maternal tissues, controlling malignant cells, or an association between microchimerism and cancer [1-3]. The changes in the micro-niches of these cells may lead them to undesirable different directions.

There can be times when things do not go as well as every physiological event. Infection with influenza during pregnancy may impair physiological fetal microchimerism and cause physiopathological microchimerism. It is known that neurodevelopment in humans is caused by the interaction of genetic, epigenetic and environmental factors. In mammals, the building blocks of brain development are completed before birth. The developmental stones underlying brain development in humans are highly susceptible to adverse effects and can be modulated by maternal markers transferred vertically during pregnancy. Observational studies show that prenatal adverse environmental effects, such as maternal stress and infections affect fetal neurodevelopment and increase the risk for neurological or psychiatric disorders in future life [4-7]. Degradation of neurodevelopmental pathways may affect cognitive and mental disorders, as well as affect future brain function and increase the risk of neurodevelopmental and psychiatric disorders in later life [8]. In pregnancy, hormones such as glucocorticoids, maternal immune cells that change in response to prenatal challenges, and cytokines pass into the fetus via the placenta. We think that maternal cytokines and hormones may also occur pathophysiological as a reaction to fetal microchimerism in the fetus. MMcCs together with hormones and cytokines may induce the epigenetic changes in the fetal brain. To date, the views on the functional role of maternal immune cells and cytokines are limited but, MMcCs

${ }^{*}$ Correspondence to: Osman Demirhan, Adana City Training and Research Hospital, Beyazevler neighborhood, 80055 street, Onsa Apartment, B-blok, 7/22, Cukurova, Adana, Turkey, Tel: 0506-0229765; E-mail: odemirhan42@gmail.com

Key words: microchimerism; fetal-maternal microchimeric cells; brain development; neurodevelopmental and psychiatric disorders; autoimmune diseases; viral disease; canser

Received: June 03, 2019; Accepted: June 21, 2019; Published: June 24, 2019 
are considered to be effective. In addition to fetal brain development of $\mathrm{MMcCs}$, it may affect postnatal cognitive performance and behavior. Recent data suggest that the brain development in response to prenatal stress challenges can be altered across several generations, independent of a genetic predisposition, supporting an epigenetic inheritance.

The adaptation processes of the fetus are very sensitive to adverse stresses such as maternal stress and / or maternal infections. In this context, chronic stress conditions affect pregnant women [9]. In addition, such negative life events can also pose a significant threat to maternal health during pregnancy. Excessive stress experienced by the mother during pregnancy may cause autism spectrum disorder (ASD) [10], depressive symptoms [11,12], anxiety, personality disorder, eating disorders [13] and attention deficit/hyperactivity disorder (ADHD) in children [6,14-16]. Besides high stress perception, maternal infection during pregnancy can interfere with fetal neurodevelopment and increase the risk for neurological dysfunctions, psychiatric disorders and ASD in the offspring [17].

In humans, healthy brain development is largely dependent on the endocrine and immunological homeostasis of the mother and fetus. Many studies have shown that maternal immune activation during pregnancy adversely affects fetal brain development at many levels. Prenatal stimuli and harmful effects including maternal stress perception and infection have been shown to interact with maternal endocrine and immunological hemostasis. At the same time, the balance between proand antiinflammatory cytokines is crucial, as neurogenesis, migration, differentiation and apoptosis are well known to be responsive to cytokine challenges. Therefore, exposure to an unbalanced cytokine response during fetal life may disrupt fetal brain development, thereby increasing the risk for neurodevelopmental disorders [18]. However, an increase in glucocorticoid concentration due to high maternal stress may adversely affect fetal brain development [19].

Cytokines, inflammation, and glucocorticoids have been also suggested to have key effects on microglia cells and neurons in the prefrontal cortex, hippocampus and amygdala of the fetal brain [17]. Similar to cytokines, it has been proposed that fetuses exposed to high concentrations of maternal glucocorticoids have long-lasting and partially sex-specific effects on brain morphology and function in rodents and humans. In these mice, decreased dendritic morphology and neuronal volume were found in the hippocampus of both genders, whereas in females, increased dendritic branching was detected only in women [20]. Moreover, a reduced expression of mGluR receptors in the hippocampus has been observed in response to prenatal stress [21-23]. However, hippocampal volume is also reduced in patients with unipolar depression, maybe as a consequence of repeated periods of major depressive disorder [24]. Some volumetric studies have found significant bilateral volume deficits in depression $[25,26]$. Others have found significantly lower volume in the right hemisphere $[27,28]$ or in the left hemisphere $[29,30]$.

Remarkably, in mouse models, brain function changes can be passed on to future generations [31], suggesting that epigenetic changes may be intergenerational. Intriguingly, how epigenetic changes induced by environmental challenges can be maintained throughout the multiple epigenetic reprogramming events physiologically occurring during reprogramming of primordial germ cells and the zygote is still greatly elusive [17]. However, to date, convincing evidence of transgenerational inheritance of epigenetic marks is only available from botany research using plants, whilst confirmation in mammals is somewhat elusive [32]. In during pregnancy, the environmental harmful effects are also subsequently leading to increased cytokines and glucocorticoids levels and potentially to altered frequencies or phenotypes of maternal microchimeric cells in the offspring.

In a recent study; the case of male with treatment-resistant schizophrenia, with predominant severe delusion and hallucination, who received bone marrow transplantation (BMT) for acute myeloid leukemia was presented. After BMT, he showed a remarkable reduction in psychotic symptoms without administration of neuroleptics. The authors put forward that the major underlying pathogenic mechanism of schizophrenia is immune dysregulation [33-38]. We think that the psychotic improvements in the patient are due to the disappearance of maternal microchimeric cells and microchimeric stem cells in the patient as a result of the curative treatment of bone marrow transplantation. It was established in radiation chimaeras more than 50 years ago that the immunological and hematological systems have a common stem cell. We believe that these common stem cells originate from fetal and maternal stem cells in this tipe patient.

If pregnant mother has a viral disease; maternal leukocytes eradicate the viral disease by passing the fetus because the immune system of the fetus is not fully developed yet. In this way, the fetus is protected from viral disease. Infectious diseases can enhance routine cell migration between mother and fetus. The mother's cell passing to protect the fetüs are involved in the construction of various tissues and organs without returning. These MMcCs that settle into the fetal tissues and do not return, there are various interpretations about their functions. We claim to be called physiopathological microchimerism. The most wellknown damage in this regard is the influence of intelligence and behavior. The strongest evidence for maternal infection increasing risk for a mental disorder in the offspring is the connection between schizophrenia and maternal respiratory infection [39,40]. An increase in the amount of viral infections and schizophrenia was found in winter and spring. Thus, the risk for schizophrenia in the offspring is increased 3-fold by infection in the second trimester [39]. Numerous animal studies demonstrate that prenatal or early postnatal infections can result in both acute and persistent neurological and behavioral abnormalities in offspring resembling autistic traits or schizophrenia $[3,40]$. Modeling this risk factor in animals, when influenza infection is induced in pregnant rodents during pregnancy, their offspring show several behavioral and histological abnormalities consistent with human mental illness [41].

We hypothesize that the MMcCs were first transformed into maternal microchimeric stem cells and then differentiated to participate in the construction of the fetal tissues and organs, and some of them were differentiated and survived as maternal microchimeric stem cells to repair future tissue damage [21]. FMcCs in the breast are not meant to contribute to the mother's health. They are matured and formatted that are reintroduced to the baby through breastfeeding. If the mother has miscarriage or abortion, the MMcCs will not be transferred to the offspring, and these cells will remain in the mother's breast tissue and will have to wait. FMcCs that do not return to the fetus will cause alloimmunity even at low density, and perhaps they will cause cancer.

McCs show immunological competence and multi-generational potential that can have important roles in cancer and specific tissue repair in autoimmune disorders and transplant rejection or graft versus host reactions. Investigators have postulated that some fetal cells escape maternal immune surveillance mechanisms and could be implicated in the pathogenesis of certain autoimmune diseases that resemble chronic GVHD responses, as seen in recipients of allogeneic grafts [42,43]. It has 
been suggested that fetal immune cells may be reactive against maternal antigens, thus triggering a graft-versus-shock reaction. Some so-called autoimmune diseases in women might be alloimmune and represent a chronic graft-versus host response attributable to transplacentally acquired fetal cells. Recent investigations show that Mc may play a role in the development of autoimmune diseases and that autoimmune diseases may not be an autoimmune disease but an alloimmune disease. The amount of FMcCs in blood and tissues of women with autoimmune disease was found to be higher than that of healthy women. The disease stages of autoimmune diseases and their healing process are also observed in psychiatric diseases. FMcCs are thought to cause maternal malignancies as well. Therefore, MMcCs could potentially operate as effectors' cells or as targets of an immune response. The previous reports show that $\mathrm{MMcCs}$ are found in more subjects with autoimmune thyroid diseases than in any other thyroid diseases $[42,44]$.

$\mathrm{MMcCs}$ are considered foreign tissues in the fetus. However, MMcCs may be providing stem cells for new cell production. During the course of MMcCs, the numbers to which they should be confined should be limited because these are the elderly cells. This limit may be exceeded several times under the influence of chronic inflammatory destruction. MMcCs may undergo apoptosis several times under the influence of inflammatory destruction and be regenerated. This destructionregeneration may also shorten the telomeres of MMcCs. After some time, under the stress of these "destruction and reconstruction", MMcCs may become cancerous. The phenomenon of FMc inspires numerous questions. First of all, we focused on does Mc play any role in carcinogenesis. Whether a F-McCs has a beneficial or detrimental effect partially depends on what kind of cell it develops into.

Pathogenesis of cancer has become a focal point of intensive research in recent years. In malign tumors, FMc is assumed to have a positive effect on tumor burden, however, some evidence shows that FMcCs may play a role in neoplastic progression. In tissues, $\mathrm{FMcCs}$ have been found in tumor sections from malignancies such as thyroid, breast, cervix, lung cancers and melanomas and have been shown to differentiate into epithelial, hematopoietic, endothelial and mesenchymal cells. However, we are only beginning to understand the role that F-MMcCs play in cancer. As a result of the mutation or microenvironmental influences, these cells may lose regulatory controls that normally keep cell proliferation and differentiation in check and thus, cancer may develop. It is intriguing to speculate that, as a consequence of genetic or epigenetic alterations or changes in their microenvironmental niche, these cells may act like cancer stem cells and give rise to tumors. The concept of pathogenic Mc was initially suggested by Nelson [45]. Some researchers have recently speculated about three new possible roles of these foreign cells in relation with cancer, which are cell cancer destruction, tissue repair, covers pathogenic and promotion of cell cancer progression [46]. Mc has been documented in autoimmune diseases, and nonautoimmune diseases, such as hepatitis $\mathrm{C}$, some tumors, including breast, thyroid, cervix, lung cancers, hematological malignancies and melanomas. For these diseases, a protective role has been hypothesized. In malignant tumors, FMc has been postulated to have a positive effect on tumor burden, although some evidence suggests that FMCs may be involved in neoplastic progression. It is suggested that $\mathrm{FMcCs}$ are present in sites of tissue injury, and may be stem cells, either recruited from marrow or having proliferated locally [47]. Sawicki hypothesized that FMcCs have tumorigenic potential and can act as cancer stem cells [48]. She described a case in which fetal cells constituted the majority of the lung tumors in a murine model. Based on these studies, it is unclear whether $\mathrm{FMcCs}$ are involved in tissue repair or contribute to tumor growth.
FMcCs have been hypothesized to be involved in chronic inflammatory responses leading to tissue damage or to participate in the repair of damaged tissue and in resistance to infections. Unfortunately, we were not able to determine the nature of these cells. Why do McCs observed in patients with cancer, but not in the controls? McCs were frequently seen in the tissues of patients. Their frequency in tumors was severalfolds higher in lung tumors than in the surrounding healthy lung tissue. FMcCs have also been shown to cluster in lung tumors in women decades after pregnancy [49].

FMcCs with hematopoietic differentiation have been postulated to have a role in destroying the tumor, whereas mesenchymal and epithelial cells could participate in repair processes. Endothelial male cells, on the other hand, could be involved in tumor progression, highlighting that a pathogenic mechanism or a detrimental role for $\mathrm{FMcCs}$ must also be considered. Indeed, the protective effect of FMc is certainly not proven, considering the high frequency of breast cancer in parous women and the marked predilection for thyroid cancer in the female sex. Median concentrations of fetal cells in peripheral blood were significantly higher in healthy women than in patients with breast cancer. FMcCs and spreading cancer cells are highly proliferative. Tumors may be loaded with FMcCs, for example, suggesting that they might help drive cancer. Breast carcinomas or melanomas have been suspected to be more severe during gestation. In particular, whether FMcCs could be able to differentiate in host tissues, participating in the maternal response to injury. The occurrence of MMcCs in tumor tissues may be related to their active involvement in the complex process of tumorigenesis, involving tumor initiation and propagation inclusive of integration into the tumor stroma [39], neoangiogenesis, facilitation of metastasis [50], induction of immune responses [3] followed by reparation of inflammation damaged tissues, as previously suggested for certain autoimmune diseases and malignancies [41]. Nevertheless, although the existence of FMcCs has been definitely shown in several studies, their role has so far only been hypothesized on the basis of associative studies and is not yet fully elucidated. We believe that microchimerism may be an important alternative explanation to the etiology of diseases.

We believe that Mc is a physiological event but, Mc may be an important alternative explanation to the etiology of diseases. Numerous studies demonstrate that prenatal or early postnatal infections can result in both acute and persistent neurological and behavioral abnormalities in offspring resembling autistic traits or schizophrenia. Further, the informations and findings indicate that pluripotent $\mathrm{McCs}$ can turn into cancer stem cells. So, we speculated that whether Mc could have a role in cancer and are just beginning to understand the new role that fetalmaternal McCs play in cancer.

\section{References}

1. Nelson JL (2009) Naturally acquired microchimerism: for better or for worse. Arthritis Rheum 60: 5-7. [Crossref]

2. Nelson JL, Gillespie KM, Lambert NC, Stevens AM, Loubiere LS, et al. (2007) Maternal microchimerism in peripheral blood in type 1 diabetes and pancreatic islet beta cell microchimerism. Proc Natl Acad Sci USA 104: 1637-1642. [Crossref]

3. Gadi VK (2009) Fetal microchimerism and cancer. Cancer Lett 276: 8-13. [Crossref]

4. Christian MA, Samms-Vaughan M, Lee M, Bressler J, Hessabi M, et al. (2018) Maternal exposures associated with autism spectrum disorder in jamaican children. $J$ Autism Dev Disord 48: 2766-2778. [Crossref]

5. Dimitrova N, Turpin H, Borghini A, Harari MM, Urben S, et al. (2018) Müller-Nix C, Perinatal stress moderates the link between early and later emotional skills in very preterm-born children: an 11-year-long longitudinal study. Early Hum Dev 121: 8-14. [Crossref] 
6. Glynn LM, Howland MA, Sandman CA, Davis EP, Phelan M, et al. (2018) Prenatal maternal mood patterns predict child temperament and adolescent mental health. $J$ Affect Disord 228: 83-90. [Crossref]

7. Vizzini L, PopovicM, ZugnaD, Vitiello B, TrevisanM, et al. (2018) Maternal anxiety, depression and sleep disorders before and during pregnancy, and preschool ADHD symptoms in the NINFEA birth cohort study. Epidemiol Psychiatric Sci 1-11. [Crossref]

8. Bale TL, Baram TZ, Brown AS, Goldstein JM, Insel TR, et al. (2010) Early life programming and neurodevelopmental disorders. Biol Psychiatry 68: 314-319. [Crossref]

9. Fisher J, Cabral de Mello M, Patel V, Rahman A, Tran T, et al. (2012) Prevalence and determinants of common perinatal mental disorders in women in low- and lower-middle-income countries: a systematic review. Bull World Health Organ 90: 139G-149G. [Crossref]

10. Walder DJ, Laplante DP, Sousa-Pires A, Veru F, Brunet A, et al. (2014) Prenatal maternal stress predicts autism traits in 61/2 yearold children: Project Ice Storm Psychiatry Res 219: 353-360. [Crossref]

11. Betts KS, Williams GM, Najman JM, Alati R (2015) The relationship between materna depressive, anxious, and stress symptoms during pregnancy and adult offspring behavioral and emotional problems. Depress Anxiety 32: 82-90. [Crossref]

12. Kingsbury M, Weeks M, MacKinnon N, Evans J, Mahedy L, et al. (2016) Stressful life events during pregnancy and offspring depression: evidence from a prospective cohort study. J Am Acad Child Adolesc Psychiatry 55: 709-716e2. [Crossref]

13. St-Hilaire A, Steiger H, Liu A, Laplante DP, Thaler L, et al. (2015) A prospective study of effects of prenatal maternal stress on later eatingdisorder manifestations in affected offspring: preliminary indications based on the Project Ice Storm cohort. Int J Eat Disord 48: 512-516. [Crossref]

14. O’Donnell KJ, Glover V, Holbrook JD, O'Connor TG (2014) Maternal prenatal anxiety and child brain-derived neurotrophic factor (BDNF) genotype: effects on internalizing symptoms from 4 to 15 years of age. Dev Psychopathol 26: 1255-1266. [Crossref]

15. Zhu P, Hao JH, Tao RX, Huang K, Jiang XM, et al. (2015) Sex-specific and timedependent effects of prenatal stress on the early behavioral symptoms of ADHD: a longitudinal study in China. Eur Child Adolesc Psychiatry 24: 1139-1147.. [Crossref]

16. Pickles A, Sharp H, Hellier J, Hill J (2017) Prenatal anxiety, maternal stroking in infancy, and symptoms of emotional and behavioral disorders at 3.5 years. Eur Child Adolesc Psychiatry 26: 325-334. [Crossref]

17. Schepanski S, Buss C, Hanganu-Opatz IL, Arck PC (2018) Prenatal Immune and Endocrine Modulators of Offspring's Brain Development and Cognitive Functions Later in Life. Front Immunol 9: 2186. [Crossref]

18. Bauer S, Kerr BJ, Patterson PH (2007) The neuropoietic cytokine family in development, plasticity, disease and injury. Nat Rev Neurosci 8: 221-232. [Crossref]

19. Solano ME, Holmes MC, Mittelstadt PR, Chapman KE, Tolosa E (2016) Antenatal endogenous and exogenous glucocorticoids and their impact on immune ontogeny and long-term immunity. Semin Immunopathol 38: 739-763. [Crossref]

20. Mychasiuk R, Gibb R, Kolb B (2012) Prenatal stress alters dendritic morphology and synaptic connectivity in the prefrontal cortex and hippocampus of developing offspring. Synapse 66: 308-14. [Crossref]

21. Laloux C, Mairesse J, Van Camp G, Giovine A, Branchi I, et al. (2012) Anxiety-like behaviour and associated neurochemical and endocrinological alterations in male pups exposed to prenatal stress. Psychoneuroendocrinology 37: 1646-58. [Crossref]

22. Zuena AR, Mairesse J, Casolini P, Cinque C, Alema GS, et al. (2008) Prenatal restrain stress generates two distinct behavioral and neurochemical profiles in male and female rats. PLOS ONE 3: e2170. [Crossref]

23. Yaka R, Salomon S, Matzner H, Weinstock M (2007) Effect of varied gestational stress on acquisition of spatial memory, hippocampal LTP and synaptic proteins in juvenile male rats. Behav Brain Res 179: 126-132. [Crossref]

24. Videbech P, Ravnkilde B (2005) Hippocampal Volume and Depression: A MetaAnalysis of MRI Studies. Am J Psychiatry 161: 11. [Crossref]

25. MacQueen GM, Campbell S, McEwen BS, Macdonald K, Amano S, et al. (2003) Course of illness, hippocampal function, and hippocampal volume in major depression. Proc Natl Acad Sci USA 100: 1387-1392. [Crossref]

26. Sheline YI, Sanghavi M, Mintun MA, Gado MH (1999) Depression duration but not age predicts hippocampal volume loss in medically healthy women with recurrent major depression. J Neurosci 19: 5034-5043. [Crossref]

27. Bell-McGinty S, Butters MA, Meltzer CC, Greer PJ, Reynolds CF III, et al. (2002) Brain morphometric abnormalities in geriatric depression: long-term neurobiological effects of illness duration. Am J Psychiatry 159: 1424-1427. [Crossref]
28. Steffens DC, Byrum CE, McQuoid DR, Greenberg DL, Payne ME, et al. (2000) Hippocampal volume in geriatric depression. Biol Psychiatry 48: 301-309. [Crossref]

29. Mervaala E, Fohr J, Kononen M, Valkonen-Korhonen M, Vainio P (2000) Quantitative MRI of the hippocampus and amygdala in severe depression. Psychol Med 30: 117125. [Crossref]

30. Bremner JD, Narayan M, Anderson ER, Staib LH, Miller HL, et al. (2000) Hippocampa volume reduction in major depression. Am J Psychiatry 157: 115-117. [Crossref]

31. Bohacek J, Gapp K, Saab BJ, Mansuy IM (2013) Transgenerational epigenetic effects on brain functions. Biol Psychiatry 73: 313-320. [Crossref]

32. Heard E, Martienssen RA (2014) Transgenerational epigenetic inheritance: myths and mechanisms. Cell 157: 95-109. [Crossref]

33. Miyaoka T, Wake R, Hashioka S, Hayashida M, Oh-Nishi A, et al. (2017) Remission of Psychosis in Treatment-Resistant Schizophrenia following Bone Marrow Transplantation: A Case Report. Front Psychiatry 8: 174. [Crossref]

34. Brown AS (2006) Prenatal infection as a risk factor for schizophrenia. Schizophr Bull 32: 200-202. [Crossref]

35. Penner JD, Brown AS (2007) Prenatal infectious and nutritional factors and risk of adult schizophrenia. Exp Rev Neurotherap 7: 797-805. [Crossref]

36. Meyer U, Feldon J, Dammann O (2011) Schizophrenia and autism: both shared and disorder-specific pathogenesis via perinatal inflammation? Pediatr Res 69: 26R-33R. [Crossref]

37. Patterson PH (2011a) Maternal infection and immune involvement in autism. Trends Mo Med 17: 389-394. [Crossref]

38. Shi L, Fatemi SH, Sidwell RW, Patterson PH (2003) Maternal influenza infection causes marked behavioral and pharmacological changes in the offspring. $J$ Neurosci 23: 297-302. [Crossref]

39. Dubernard G, Aractingi S, Oster M, Rouzier R, Mathieu MC, et al. (2008) Breas cancer stroma frequently recruits fetal derived cells during pregnancy. Breast Cancer Res 10: R14. [Crossref]

40. Nguyen Huu S, Oster M, Avril MF, Boitier F, Mortier L, et al. (2009) Fetal microchimeric cells participate in tumour angiogenesis in melanomas occurring during pregnancy. $\mathrm{Am}$ J Pathol 174: 630-637. [Crossref]

41. Sawaya HHB, Jimenez SA, Artlett CM (2004) Quantification of fetal microchimeric cells in clinically affected and unaffected skin of patients with systemic sclerosis. Rheumatology 43: 965-968. [Crossref]

42. Lee ESM, Bou-Gharios G, Seppanen E, Khosrotehrani K, Fisk NM (2010) Fetal stem cel microchimerism: natural-born healers or killers? Mol Hum Reprod 16: 869-878. [Crossref]

43. Klintschar M, Schwaiger P, Mannweiler S, Regauer S, Kleiber M (2001) Evidence of fetal microchimerism in Hashimoto's thyroiditis. J Clin Endocrinol Metab 86: 2494 2498. [Crossref]

44. Ando T, Imaizumi M, Pritsker A, Davies TF (2001) Identification of feta microchimerism in the human thyroid. Abstract OR23-1. 83 ${ }^{\text {rd }}$ Annual Meeting of the Endocrine Society 20-23

45. Ando T, Imaizumi M, Graves PN, Unger P, Davies TF (2002) Intrathyroidal fetal microchimerism in Graves' disease. J Clin Endocrin Metab 87: 3315-3320. [Crossref]

46. Nelson JL (1996) Maternal-fetal immunology and autoimmune disease: is some autoimmune disease auto-alloimmune or allo-autoimmune? Arthritis Rheum 39: 191946. [Crossref]

47. Galofré zjc, Duntas LH, Premawardhana LD, Davies TF (2012) Advances in Graves Disease. J Thyroid Res 809231. [Crossref]

48. O’Donoghue K, Chan J, de la Fuente J, Kennea N, Sandison A, et al. (2004) Microchimerism in female bone marrow and bone decades after fetal mesenchymal stem cell trafficking in pregnancy. Lancet 364:179-182. [Crossref]

49. Sawicki JA (2008) Fetal microchimerism and cancer. Cancer Res 68: 9567-9569. [Crossref]

50. Nelson JL (2001) Microchimerism: expanding new horizon in human health or incidental remnant of pregnancy? Lancet 358: 2011-2012. [Crossref]

Copyright: (C2019 Davies D. This is an open-access article distributed under the terms of the Creative Commons Attribution License, which permits unrestricted use, distribution, and reproduction in any medium, provided the original author and source are credited. 